\title{
REACTIVE HYPEREMIA INDEX AND DETECTION OF ENDOTHELIAL DYSFUNCTION IN HIGH RISK CHILDREN
}

\author{
P. Jehlicka ${ }^{1}$, M. Huml ${ }^{1}$, T. Votava ${ }^{1}$, R. Pomahacova ${ }^{1}$, L. Trefil ${ }^{2}$, J. Kobr ${ }^{1}$ \\ ${ }^{1}$ Paediatrics, ${ }^{2}$ Clinical Biochemistry, Medical University Hospital of Pilsen, Plzen, Czech Republic
}

Objective: To evaluate Reactive Hyperemic Index (RHI) as an indicator of endothelial function (EF) in children with type 1 diabetes mellitus (T1DM) and in children following treatment for acute lymphoblastic leukemia (ALL) in comparison with healthy controls (HC). RHI was further correlated with anthropometric and biochemical parameters.

Research design and methods: 54 eligible study participants were enrolled in the study (14 T1DM (16 $\pm 2.2 \mathrm{yrs})$ and 28 ALL patients (14 $\pm 3.4 \mathrm{yrs})$ matched with $12 \mathrm{HC}(16 \pm 1,7 \mathrm{yrs}))$. As part of new non-invasive plethysmographic technique, an Endo-PAT2000 ${ }^{\circledR}$ recorder was used for the determination of RHI by measuring postocclusive endothelium-dependent changes in vascular tone (PAT) in subjects fingertips.

Results: Significantly lower RHI were revealed in T1DM and ALL patients in comparison with HC $(1.50 \pm 0.56,1,57 \pm 0.50$ and $1.96 \pm 0.62 ; \mathrm{p} \leq 0.05$ respectively), implying impaired endothelial-dependent dilation. No association was revealed between RHI and anthropometric parameters, arterial blood pressure or glycated haemoglobin in both T1DM and ALL groups.

Conclusion: Detection of endothelial dysfunction using RHI in T1DM children is in concordance with results of previously published studies. Low RHI in ALL children supports our hypothesis of endothelial dysfunction in this group. Our study demonstrated that a non-invasive method such as RHI is a promising future prospect for the assessment of EF in children with high risk of premature atherosclerosis.

This work was supported by the Charles University Student Research Project SVV-2010-260805 